\title{
Effective laser driven proton acceleration from near critical density hydrogen plasma
}

\author{
ASHUTOSH SHARMA $^{1}$ AND ALEXANDER ANDREEV ${ }^{1,2}$ \\ ${ }^{1}$ ELI-ALPS, Szeged, Hungary \\ ${ }^{2}$ Max-Born Institute, Berlin, Germany \\ (RECEIVED 10 September 2015; ACCEPTED 11 January 2016)
}

\begin{abstract}
Recent advances in the production of high repetition, high power, and short laser pulse have enabled the generation of highenergy proton beam, required for technology and other medical applications. Here we demonstrate the effective laser driven proton acceleration from near-critical density hydrogen plasma by employing the short and intense laser pulse through three-dimensional (3D) particle-in-cell (PIC) simulation. The generation of strong magnetic field is demonstrated by numerical results and scaled with the plasma density and the electric field of laser. 3D PIC simulation results show the ring shaped proton density distribution where the protons are accelerated along the laser axis with fairly low divergence accompanied by off-axis beam of ring-like shape.
\end{abstract}

Keywords: Laser-plasma interaction; Magnetic vortex acceleration; Particle-in-cell simulation; Proton beam

\section{INTRODUCTION}

Beams of high-energy ions and protons could be produced by directing a high power ultra-short laser pulse onto a thin target, as investigated in 2000 by Snavely et al. (2000). Acceleration takes place at the target-vacuum interfaces where laser-accelerated relativistic electrons form a dense electron plasma sheath (field $\sim \mathrm{TV} / \mathrm{m}$ ) that ionizes surface atoms and accelerates ions in the target normal. These beams have extreme laminarity, ultra-short duration, and high particle number per bunch that distinguish them from beams produced by conventional sources (e.g., accelerators). In particular the medical applications of such beams give rise to the field of radiation therapy (Loeffler\& Durante, 2013). Recent progress in generating the high-energy $(>50 \mathrm{MeV})$ ions from intense laser-matter interactions $\left(10^{18}-10^{21} \mathrm{Wcm}^{-2}\right.$; (Hatchett et al., 2000; Snavely et al., 2000; Wilks et al., 2001; Borghesi et al., 2004; Fuchs et al., 2006; Hegelich et al., 2006; Robson et al., 2007) has opened up new areas of research, with applications in radiography (Mackinnon et al., 2006), oncology (Bulanov \& Khoroshkov, 2002), astrophysics (Baraffe, 2005), imaging (Fritzler et al., 2003), high-energy-density physics (Dyer et al., 2008), and ion-proton beam fast ignition (Roth

Address correspondence and reprint requests to: Ashutosh Sharma, ELIALPS, Szeged, Hungary. E-mail: ashutosh.sharma@eli-alps.hu et al., 2001; Key et al., 2006a, b). The fast recent progress has hinted that the extreme parameters of extreme light infrastructure will allow the production of ultra-high-energy ions ( $\mathrm{GeV}$ and beyond) which will open the door to future unique applications like time and space resolved radiography of dense matter (Borghesi et al., 2010), injectors study for medical applications (Muramatsu and Kitagawa, 2012) for ion beam physics (Hoffmann et al., 2005).

Recent development (Nickles et al., 2007; Daido et al., 2012; Jung et al., 2015) in laser driven ion acceleration from overdense plasmas demonstrated stimulated mechanisms. Target-normal sheath acceleration (TNSA) (Wilks et al., 1992; Cowan et al., 2004), radiation pressure acceleration (RPA) (Esirkepov et al., 2004; Macchi et al., 2005; Robinson et al., 2009; Palmer et al., 2011; Yao et al., 2014), shock wave acceleration (Silva et al., 2004; Haberberger et al., 2012; Fiuza et al., 2013) and relativistic transparency regime (Henig et al., 2009; Yin et al., 2011; Jung et al., 2013; Roth et al., 2013) are the novel mechanisms which are the center of experiments and theoretical investigations.

The high-energy ion acceleration from near critical density target is also of high relevance because recent analytical and simulation study reported high-energy protons. Matsukado et al. (2003) investigated the role of prepulse in acceleration of protons from thin foil. In their research it was shown that initially prepulse evaporate the irradiated region of thin foil, 
thereafter the main pulse interacting with the underdense plasma generates the electrostatic field due to magnetic field expansion and hence accelerate the ions. Bulanov et al. (2010a) investigated the scaling laws and optimal conditions for proton acceleration by the magnetic vortex mechanism (MVA) in near critical density targets. Nakamura et al. (2010) also derived the energy scaling of ions by MVA using the particle-in-cell (PIC) simulations.

Recently Gu et al. (2014) investigated the bunch of protons of maximum energy $1 \mathrm{GeV}$ using the 2.5D PIC simulation where protons experience multi acceleration mechanism [at the rear surface of target the protons are accelerated by TNSA and MVA, and later on by the long range breakout afterburner acceleration (Yin et al., 2006, 2011) during the whole acceleration process]. Kawata et al. (2014) discussed the compact and controllable laser baser ion accelerator by using a solid target with a fine sub-wavelength structure or a near critical density gas plasma. The energy efficiency from laser to ion is improved by employing such hybrid target. The ion acceleration is also demonstrated using the cluster-gas target with an ultra-short laser pulse. The PIC simulation study by Fukuda et al. (2009) revealed the production of high-energy ions at the rear side of near-critical plasma target, due to the formation of dipole vortex structure at rear edge. Using an intense circularly polarized laser, Gao et al. (2012) demonstrated efficiently the acceleration of protons from a foam-Carbon foil target. Recently Gauthier et al. (2014) have shown a promising approach to accelerate protons to high-energies using the near-critical target. The simulation study explores the relevance of density gradient of plasma target on acceleration process. Another interesting mechanism is reported by Bake et al. (2012) where the proton acceleration is demonstrated via combined mechanism of RPA and plasma bubble field. By 2D PIC simulations the energy enhancement of protons bunch is shown by using the background plasma with negative density gradient. 3D PIC simulation results reported by Lemos et al. (2012) were confirmed in an experiment conducted at University of California, Los Angeles (UCLA). In their experimental study few $\mathrm{MeV}$ energetic ions were observed in an underdense plasma by employing a $50 \mathrm{fs} / 5 \mathrm{TWs}$ Ti:Sapphire laser.

The most stable and well understood mechanism so far is the TNSA, which usually requires long pulse duration in order to reach high cut-off energy. Since at a few laboratories the ultra-short laser pulses of high repetition rate are in the center of interest, we have to consider different mechanisms, especially to avoid debris problem. The scheme of interest may be MVA, which is more efficient in near-critical density plasma.

We investigated in this 3D PIC simulation study the acceleration of protons from the near critical density hydrogen target using the ultra-short (17 fs) and high power [in petawatt (PW) regime] laser, which is not explored in past (Amitani et al., 2002) through the 3D simulations. We further explored the spatial distribution of high-energy protons which are generated via a composite acceleration mechanism of MVA and post-acceleration by the rest-over laser field itself. The 3D simulations are performed in this research to suit the experimental conditions for ion acceleration application, to employ the high repetition PW laser facility of ELI-ALPS (http://www.eli-hu.hu/). 3D simulation is also of relevance for MVA mechanism of ion acceleration because the magnetic vortex structure is a $3 \mathrm{D}$ entity, where fast electrons propagate inside the channel and the return current flows along the channel wall, which accompanies the radial electric field and azimuthal magnetic field. Thus it is desired to model the 3D simulation to investigate the effective proton acceleration utilizing the MVA mechanism, which can be advantageous over $1.5 \mathrm{D}$ or $2 \mathrm{D}$ simulation. We reported the simulation results in this study which delineate the high-energy protons with ring shaped density distribution at the plasma-vacuum interface.

\section{3D PIC SIMULATION RESULTS}

The regime of laser ion acceleration from near critical density gas targets can be realized when the laser pulse propagates through a near critical density target that is much longer than the pulse itself and the pulse forms a density channel. In MVA regime, when a tightly focused laser pulse interacts with the near-critical density plasma, the ponderomotive force of the laser expels electrons and ions in the transverse direction, forming the electron and ion density channel. A portion of the electrons are accelerated in the direction of laser pulse propagation by the longitudinal electric field. The motion of these electrons generates a magnetic field, which circulates in the channel around the propagation axis. Upon exiting the channel, the magnetic field expands into vacuum and the electron current is dissipated. Some of the electrons leave the plasma channel while few of them return to sustain the magnetic field on the rear side of plasma-vacuum interface. The magnetic field displaces the electron component of plasma with regard to the ion component and a strong quasi-static electric field is generated that can both accelerate and collimate the ions. For optimum acceleration [Bulanov et al. $(2010 a, b)$ ] in case of MVA, the laser spot size should match the size of the self-focusing channel in order to avoid filamentation. The laser pulse energy should be depleted near the target rear to create the dipole vortex at the exit side of the channel and it is not wasted to transmission.

The effectiveness of the MVA mechanism requires the efficient transfer of laser energy to the fast electrons in the plasma which are accelerated in the plasma channel along the laser propagation direction. Thus the optimal condition for efficient proton acceleration can be estimated by equating laser pulse energy in plasma waveguide to the energy of electrons. The optimum plasma length $(l)$ can be estimated from the assumption that all laser energy is transferred to the electrons in the plasma channel. If each electron has an average energy of $a_{\mathrm{L}} m_{\mathrm{e}} c^{2}$, the optimum length can be written as 
(Bulanov et al., 2010b) $l=a_{\mathrm{L}} c \tau\left(n_{\mathrm{c}} / n_{\mathrm{e}}\right) K$, where $K$ is the geometry constant ( $K$ is 0.1 in 2D case and 0.074 in 3D case) and the laser pulse amplitude $\left(a_{\mathrm{L}}\right)$, can be determined by the laser power $(P)$ and plasma density $\left(n_{\mathrm{e}}\right)$ as $a_{\mathrm{L}}=\left(8 \Pi\left(P_{0} / P_{\mathrm{c}}\right)\right.$ $\left.\left(n_{\mathrm{e}} / n_{\mathrm{c}}\right)\right)^{1 / 3}$.

Based on the theoretical approach (as outlined previously), we demonstrate here through the 3D PIC simulations the acceleration of protons by employing the ELI-ALPS high-field (HF) laser interaction with the near critical density hydrogen plasma. The incident laser parameters are chosen comfortably within the capabilities of ELI-ALPS facility. The expected parameter regime for ELI-ALPS HF laser is as follows: Laser wavelength $\lambda_{\mathrm{L}}=800 \mathrm{~nm}$, laser energy $\varepsilon_{\mathrm{L}}=$ $34 \mathrm{~J}$, and pulse duration $t_{\mathrm{L}}=17 \mathrm{fs}$. We consider here the interaction of a linearly polarized laser pulse with the plasma target. The laser pulse considered here is Gaussian in space and time where the beam radius is $r_{\mathrm{b}}=1.042 \mu \mathrm{m}$ obtained by focusing lens of $F=2$. The tightly focused beam will initially diverge in the plasma and will expel electrons to form a plasma channel, then after the divergence of laser will stop and the most of the laser energy in plasma channel will be transferred to plasma electrons to accelerate it. The plasma target proposed here is hydrogen of density $n_{\mathrm{i}}=n_{\mathrm{e}}=$ $5.22 \times 10^{21} \mathrm{~cm}^{-3}$ (three times of the critical density $n_{\mathrm{c}}$ ) and the optimum thickness of plasma target is $l=25 \mu \mathrm{m}$, to utilize the maximum laser energy transfer to plasma electrons [see (1)]. 3D PIC simulations were carried out using the fully relativistic electromagnetic code PIConGPU (Burau, 2010; Bussmann et al., 2013). The boundary conditions are periodic throughout the simulation. We considered here the simulation box of dimension $10 \times 200 \times 20 \mu^{3}$ corresponding to the grid size $256 \times 5120 \times 512$ with cell size of $40 \mathrm{~nm}$. The time step is 66.7 as. The laser pulse (polarization of laser field is along $x$-axis) incidents on plasma along the $y$-axis in $X Z$ plane. The plasma target starts at $z=0.0 \mu \mathrm{m}$ and terminates at $25.0 \mu \mathrm{m}$. The 3D simulation performed in this study limits to the simulation size corresponding to the available graphical processing units (GPUs) computing facility. We considered here $n_{\mathrm{i}}=3 n_{\mathrm{c}}$ to minimize the optimal plasma channel length and limit 3D simulations. In our simulations we considered intensities of the order of $10^{23} \mathrm{~W} / \mathrm{cm}^{2}$ or smaller so we did not incorporate the radiation reaction force (see Gao et al., 2012) along with the Lorentz force for proton acceleration.

A liquid hydrogen jet [Kühnel et al. (2011)] might be an interesting option for MVA to obtain the near critical density plasma in laboratory with ultra-intense femtosecond pulses because, besides allowing the acceleration of protons, being a "continuously flowing" target it would allow for high repetition rate operation, which is of high relevance for applications with high repetition PW laser.

The PIC simulation results (as shown by Fig. 1), summarizes the laser pulse penetration through the near critical density hydrogen plasma and consequently the generation of magnetic field at the plasma-vacuum interface.

The generation of magnetic field can be illustrated in the following manner. The relativistic electrons accelerated within the plasma channel, follow the laser pulse and exit from the rear side of the plasma channel as the pulse exist at the plasma-vacuum interface. The fast electrons leave the plasma and then return back into it under the influence of an unneutralized electric charge. The electrons form a toroidal vortex in 3D geometry (dipole vortex in 2D case) due to such motion and the electric current of vortex generates a quasi-static magnetic field. Thus in case of homogeneous plasma with sharp plasma vacuum boundary at both ends (front and rear) of plasma channel, the magnetic field produced by fast electron beam expands along the plasma slab boundary. The rapid variation in magnetic field due to the vortex motion produces a strong quasi-static electric field. The ions are accelerated to high-energies due to the quasistatic electric field at plasma-vacuum interface. The magnitude of the magnetic field can be estimated by employing the Ampere's law, $B \approx 4 \Pi \eta n_{\mathrm{e}} e r_{\mathrm{ch}}$ where $r_{\mathrm{ch}}$ is the plasma channel radius which is equal to the radius of focused laser beam. We further simplified the magnetic field to scale it with initial laser-plasma parameter and obtained as,

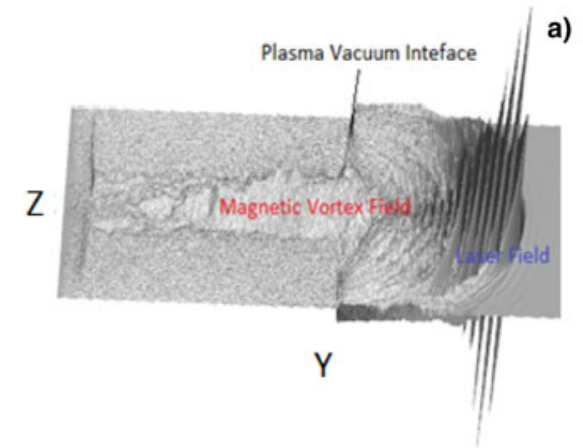

a)

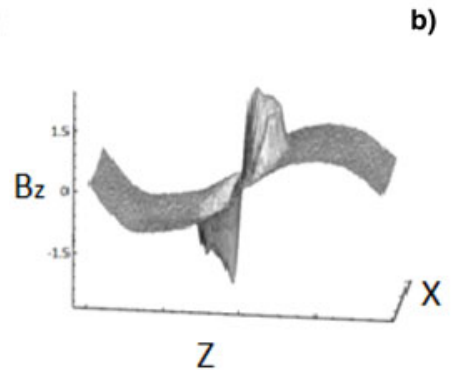

b)

c)

Fig. 1. (a) The distribution of the magnetic field of vortex structure followed by the laser magnetic field, as the laser pulse channels inside the hydrogen plasma, (b) the variation of magnetic field at plasma-vacuum interface in $X Z$ plane, (c) scaling of magnetic field with the incident laser power, analytical result (black solid line), and simulation results (red dot); at time $t=0.2$ psec. 
(in terms of Tesla). The magnitude of magnetic field (using $\eta=0.6$ taken from the simulation) is $\sim 20.0 \times 10^{4} \mathrm{~T}$, which is close to the simulation results as shown by Figure 1c. Thus the magnetic field (1) increases with the focused laser field and the plasma density, and consequently the strong magnetic field can be generated in laboratory by utilizing the high power laser interaction with the near-critical plasma. The evolution of the magnetic field depends dominantly on the scale on which the plasma density varies. In case of an inhomogeneous plasma with a density gradient at plasma-vacuum interface, the magnetic vortex moves down the density gradient and expands in forward direction due to the decrease in plasma density and in lateral direction due to force acting on the vortex in the $\nabla n X \Omega$ direction (Nycander and Isichenko, 1990). Due to the forward and lateral expansion of magnetic vortex structure the electron density in the current filament decreases to a value where the condition of charge quasi-neutrality fails to hold. Consequently the current filament undergoes a Coulomb expansion and leads the vanishing of magnetic field. The abrupt decrease in magnetic field induces a strong electric field which accelerates the ion beam to high-energies. PIC simulation studies (Bulanov et al., 2005; Nakamura et al., 2010) of high power laser interaction with gas target have shown the relevance of plasma density gradient for ion acceleration, however in this study we focus the ion acceleration utilizing the ultra-short-ultra-intense laser pulse interacting the near critical density plasma with sharp plasma-vacuum boundary.

We show the evolution of electron and ion density in Figure 2 at $200 \mathrm{fs}$, when the laser pulse exits from the plasma channel. A strong wakefield of the order of several $\mathrm{GV} / \mathrm{cm}$ is generated in the plasma, as the ultra-intense laser propagates in the near critical density plasma. The bunch of trapped electrons is then accelerated to $100 \mathrm{~s}$ of $\mathrm{MeV}$ along the channel axis. As fast electron beam ejects out of the plasma into vacuum, the most energetic electrons escapes, forming a plasma potential barrier, which prevents further acceleration of low energy electrons.

As these electrons are pulled back into plasma (return current), it generates a magnetic vortex field of magnitude

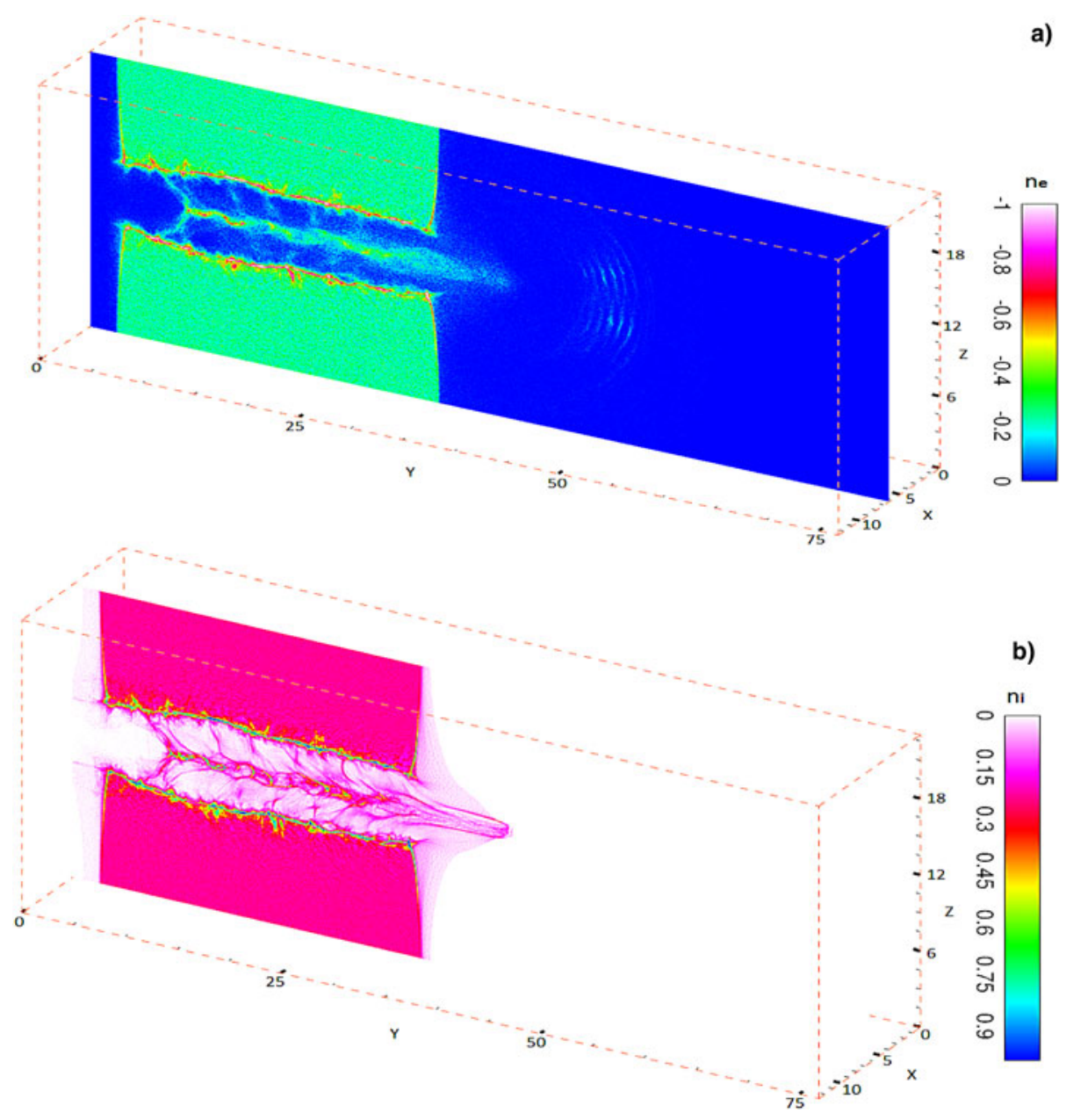

Fig. 2. Evolution of (a) electron density $\left(n_{\mathrm{e}}\right)$ and (b) ion density $\left(n_{\mathrm{i}}\right)$ at time $200 \mathrm{fs}$, after pulse exits the plasma channel. The electron and ion densities are normalized to relativistic modified critical plasma density $n_{\mathrm{cr}}=\sqrt{\gamma} n_{\mathrm{e}}$ where $\gamma$ is the relativistic factor (here $\gamma \approx 12$ ) as its variation is shown by the color bar. The $X, Y$ and $Z$-axis are shown in units of laser wavelength. 


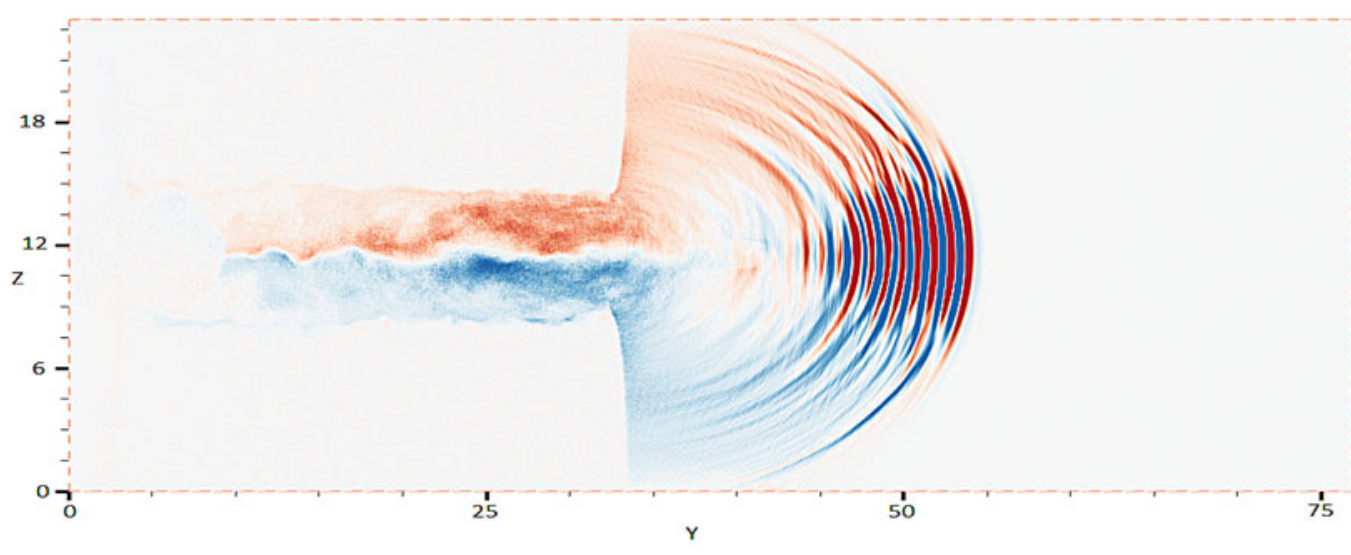

a)

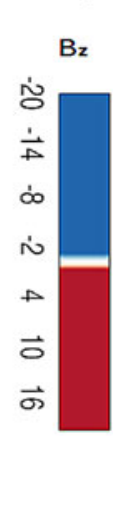

b)

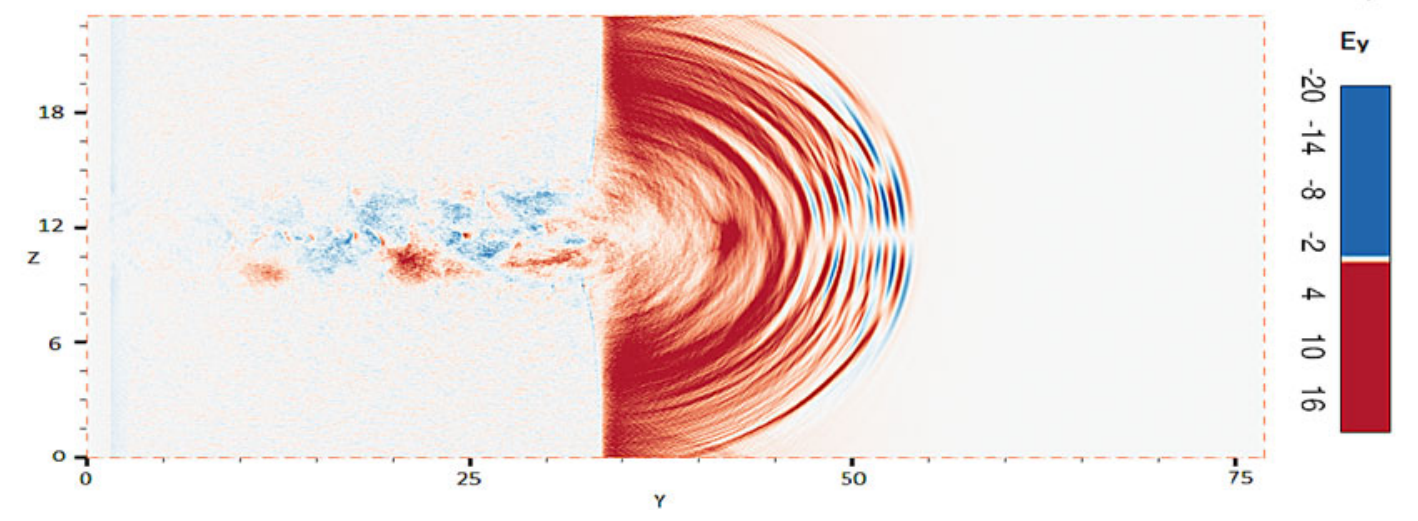

Fig. 3. Evolution of (a) magnetic vortex field ( $\left.\mathbf{B}_{z}\right)$ and (b) longitudinal electric field ( $\mathbf{E}_{\boldsymbol{y}}$ ) at (200 fs) just after pulse exits the plasma channel. The color bar shown for $\mathbf{B}_{z}$ is expressed in units of $8.52 \times 10^{4} \mathrm{~T}$ and for $\mathbf{E}_{\boldsymbol{y}}$ in units of $25.5 \mathrm{TV} / \mathrm{m}\left(E_{\mathrm{L}} \sim 500 \mathrm{TV} / \mathrm{m}\right)$. The $Y$ and $Z$-axis are shown in units of laser wavelength. The dark red and blue lines ahead of $\mathbf{B}_{z}$ and $\mathbf{E}_{\boldsymbol{y}}$ correspond to the laser field.

several megagauss (as shown in Figure 3a). The expansion of the magnetic field produces a longitudinal inductive electric field (by Faraday's Law), which accelerates the protons further. The longitudinal electric field (as shown in Fig. 3b) attains the magnitude of the order of $\mathrm{TV} / \mathrm{m}$.

The ion filament maintains over long distance because of dominant nature of focusing longitudinal field over the diverging magnetic vortex field. This electric field accelerates and collimates protons from the thin proton filament which is formed along the propagation channel. Figure 4 shows the longitudinal and transverse proton momentum along the propagation direction ( $Y$-axis) at different time instant to demonstrate the acceleration of protons at the rear side of plasma target in vacuum.

Figure $4 \mathrm{a}, \mathrm{c}$ shows the acceleration of protons at time instant $0.2 \mathrm{psec}$ where protons are accelerated to high energy from longitudinal electric field generated due to the expansion of magnetic field. Figure $4 c$, d further explain the extended acceleration of protons at time instant $0.53 \mathrm{psec}$, since as time advances the magnetic field dissipates and the acceleration of proton is due to the energy transfer from leading electrons through the rest-over laser field. The electrons and protons accelerated along the $Y$-axis are expelled by the laser field in transverse direction and the transverse expansion is favored because of coulomb explosion of particles at axis. From the phase space data (as shown in Fig. 4) one can obtain the proton angular distributions. For a specific energy range, the distribution can be calculated by calculating the number of particles going in a given angle found from

$$
\theta=\cos ^{-1}\left(p_{y} / \sqrt{p_{x}^{2}+p_{y}^{2}+p_{z}^{2}}\right)
$$

The energy spectrum of the accelerated proton bunch which is propagating close to the axis, shown in Figure 5 at time $t=$ $0.2 \mathrm{psec}$ (a) and $0.67 \mathrm{psec}(\mathrm{b})$. The maximum cut-off energy, we obtain in this case is around $1.1 \mathrm{GeV}$ at $0.67 \mathrm{psec}$. In the inset we show the energy spectrum of all protons, which are propagating along laser direction at different angle. The longitudinal momentum of protons is maintained from $t=$ $0.2 \mathrm{ps}$ to $0.67 \mathrm{psec}$ since the responsible factor for their acceleration is the longitudinal electric field.

We studied further the proton density (energy) at different time instant during the acceleration process (as shown in Fig. 6), after the laser field exits from the plasma channel. At time $167 \mathrm{fs}$ when the ions form thin filament, they are accelerated to energy of $0.16 \mathrm{GeV}$ and at time $200 \mathrm{fs}$ the ions 


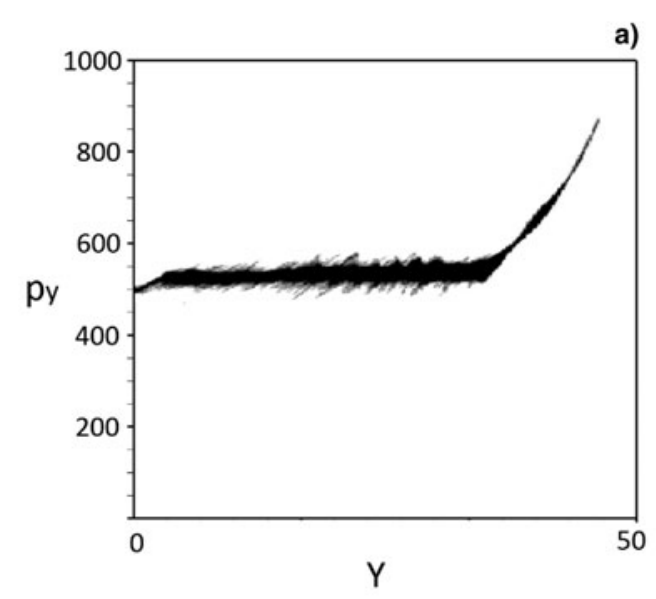

a)
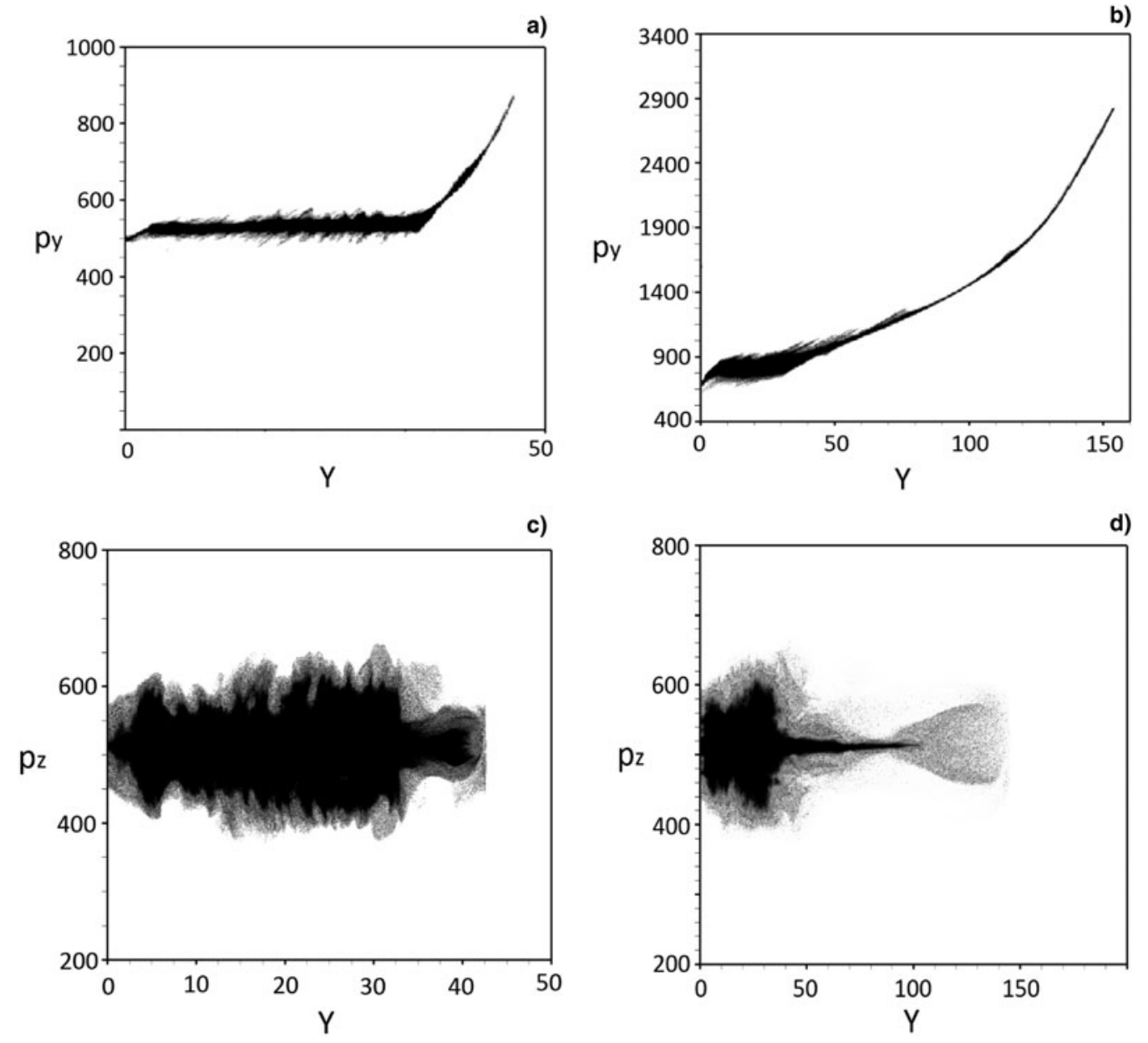

Fig. 4. Longitudinal momentum $\left(p_{y}\right)(\mathrm{a}, \mathrm{b})$ and transverse momentum $\left(p_{z}\right)(\mathrm{c}, \mathrm{d})$ of accelerated protons along the propagation direction $(Y$-axis) at time instant $(\mathrm{a}, \mathrm{c}) 0.2 \mathrm{psec}(\mathrm{b}, \mathrm{d}) 0.53 \mathrm{psec}$. The $Y$-axis is shown in units of laser wavelength $(0.8 \mu \mathrm{m})$ and longitudinal and transverse proton momentum is expressed in units of $m_{\mathrm{e}} c$.

gain energy $0.35 \mathrm{GeV}$ due to the strong longitudinal electric field produced due to time varying magnetic field. The ring shaped proton distribution (as shown in Fig. 6b) can be explained as an expansion of proton filament, which is formed due to ponderomotive expulsion of electrons from the region which is having a dimension of the order of the laser spot diameter. In Figure 6c we show the angular distribution of protons, which indicates that, the high energy
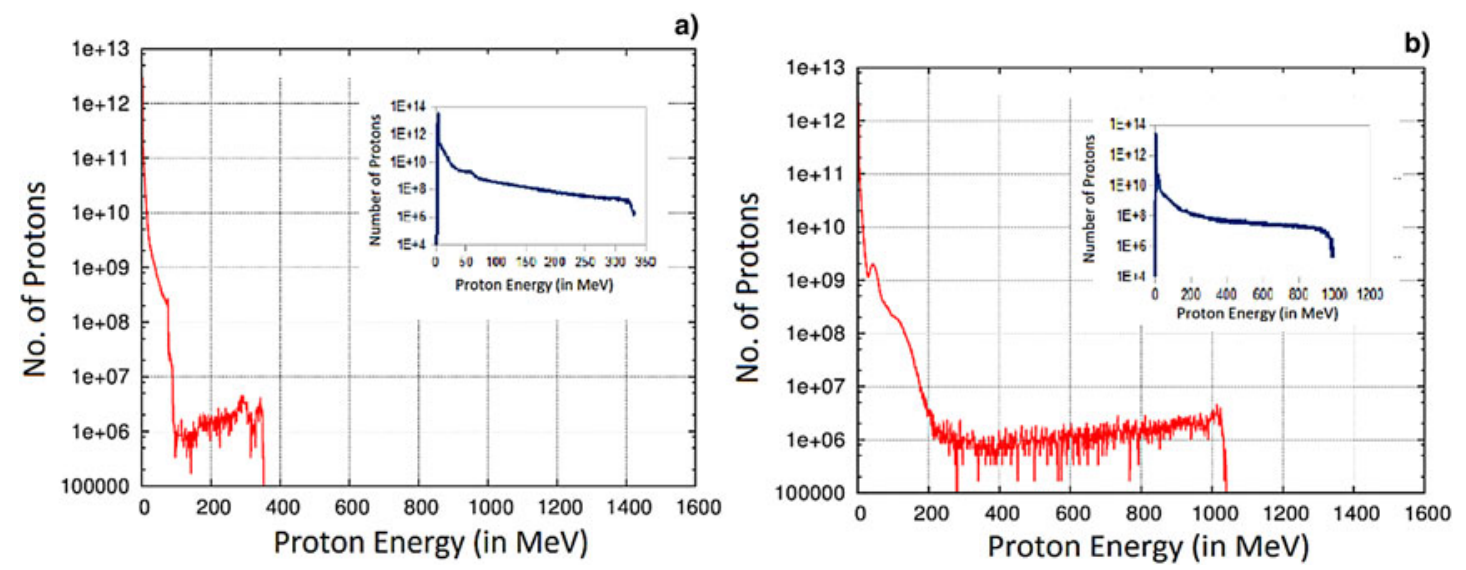

Fig. 5. Energy distribution of protons propagating close $\left(-5^{\circ}\right.$ to $\left.+5^{\circ}\right)$ to axis at $0.2 \mathrm{psec}$ (a) and $0.67 \mathrm{psec}$ (b). Inset plot: Proton energy distribution while considering the all accelerated protons. 
a)

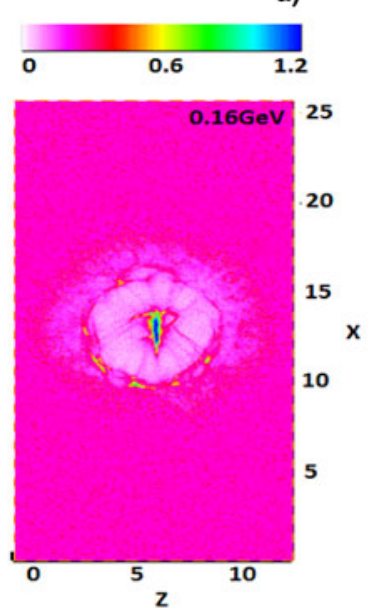

b)

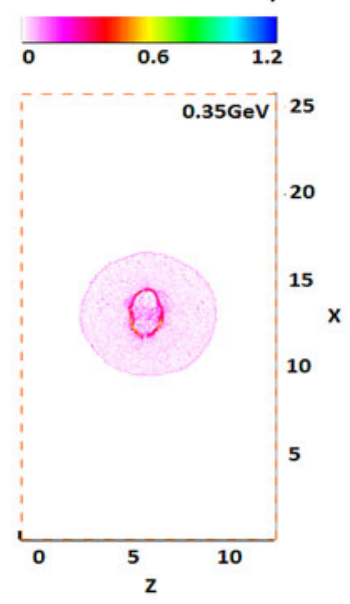

c)

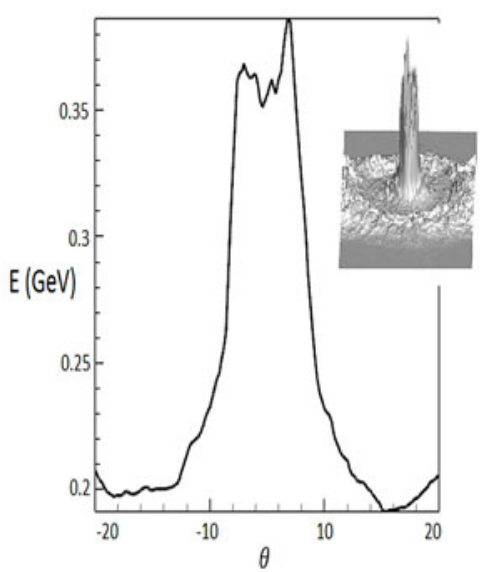

Fig. 6. The proton density distribution corresponding to different maximum proton energy (at different time instant $t=(\mathrm{a}) 0.17$ psec, (b) 0.2 psec corresponding to propagation direction at $y=$ (a) $26 \mu \mathrm{m}$ and (b) $48 \mu \mathrm{m}$. (c) The angular distribution of protons at 0.2 psec, where inset shows 3D view of proton energy distribution. The color bar corresponds to ion density normalized with critical plasma density. In (a) the pink background corresponds to unperturbed proton density since plot (a) corresponds to plasma-vacuum interface at the rear side of the plasma channel.

protons are well collimated with the small divergence angle. It is worth to note that the results shown in Figure 5 correspond to the protons propagating close to the ring and the results shown in Fig. 5a is corresponding to Fig. $6 \mathrm{~b}$ at 0.2 psec.

The generation of magnetic field at plasma-vacuum interface is shown in Figure 3a and subsequently the quasi-static electric field accelerating protons in Figure $3 b$. However the simulation results (see Fig. 3a, b) also show the electric and magnetic field of transmitted laser field, which is not completely depleted in the plasma channel. Thus the protons accelerated by the MVA mechanism at plasma-vacuum interface may also get influenced by the transmitted laser fields. As the pulse exits the plasma channel (as shown in Fig. 2a), the leading hot electrons propagate in vacuum and form a bow in front of the protons. The magnetic field upon exiting the channel pushes further protons in respect of electrons and collimated proton bunch follows the leading electrons (co-moving with the laser field). Thus leading electrons transfer their energy to proton through the charge separation field and electrons are continuously getting energy from the laser pulse, which extends the acceleration length to obtain the high proton energy. We explore further the mechanism of acceleration of proton by investigating the evolution of magnetic field (Fig. 7a) and longitudinal electric field (Fig. 7b) which is responsible for the acceleration of electrons and protons. At initial acceleration stage the ions gain energy from the longitudinal electric field which is produced by the expansion of magnetic field. The magnetic field is maintained for $106 \mathrm{fs}$ after the laser pulse exits the plasma channel (as shown in Fig. 7a, since the magnetic field of vortex structure exits along the $Y$-axis for distance $32 \mu \mathrm{m}$ starting from 30 to 70 laser wavelength, which corresponds to $106 \mathrm{fs}$ time period). In this time period the protons gain energy $670 \mathrm{MeV}$. After this time the acceleration continues at slower rate due to the expansion of magnetic field. The post acceleration mechanism which is responsible after this time is due to longitudinal electric field between electrons, which is co-moving with laser field (as shown in Fig. 7c, d) and the accelerated protons. The proton energy density (Fig. 7c) and electron energy density (Fig. 7d) at time $0.53 \mathrm{psec}$ is shown to reveal the post acceleration process along the propagation axis. Thus as time advances the magnetic field dissipates and the dominant acceleration of proton is due to the energy transfer from leading electrons.

Following the model of Bulanov et al. (2015) the maximum proton energy from MVA can be written as $E_{\mathrm{P}}=\eta \gamma_{\mathrm{e}}^{2}$ $a_{\mathrm{L}} m_{\mathrm{e}} c^{2}$ where the proton energy $\left(E_{\mathrm{P}}\right)$ scales with the laser power as $P^{0.67}$ and $\gamma_{\mathrm{e}}=(\sqrt{2 / 1.84})\left(2 P / K P_{\mathrm{c}}\right)^{1 / 6}\left(n_{\mathrm{cr}} / n_{\mathrm{e}}\right)^{1 / 3}$ is the Lorentz factor of accelerated bulk electrons (which can be obtained from the condition that electron velocity is equal to the group velocity of an electromagnetic pulse propagating in a waveguide).

In Figure 8, we show the dependence of maximum proton energy on the laser power for the optimized value of lasertarget parameter. We obtained the scaling of proton energy with the laser power as $E_{\mathrm{P}} \sim P^{0.67}$, which is consistent with the model result.

The proton energy scaling demonstrates that few hundred $\mathrm{MeV}-\mathrm{GeV}$ protons can be obtained by employing the high power laser (from $100 \mathrm{TW}-2 \mathrm{PW}$ ) interaction with the hydrogen near critical density plasma. The $3 \mathrm{D}$ simulation results in the considered regime show the laser-to-proton conversion efficiency $\sim 1 \%$ (for protons with energy $>100 \mathrm{MeV}$ and cut-off energy $\sim 1 \mathrm{GeV})$. The highest TNSA proton energy $(\sim 70 \mathrm{MeV})$ has been obtained by using single shot $80 \mathrm{~J}$ laser pulse and specially designed targets, where the conversion efficiency (for proton $>4 \mathrm{MeV}$ energy) was also about $1 \%$ [Gaillard et al. (2011)]. In our case even 
a)

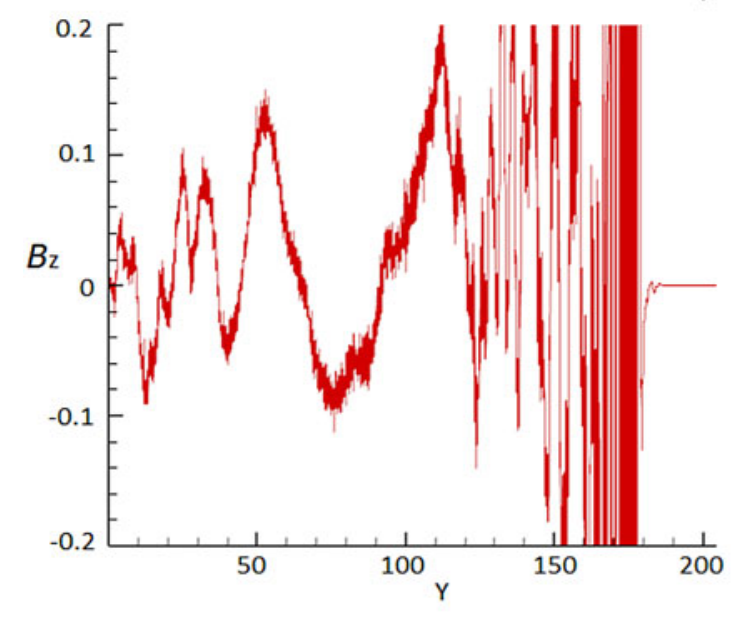

c)

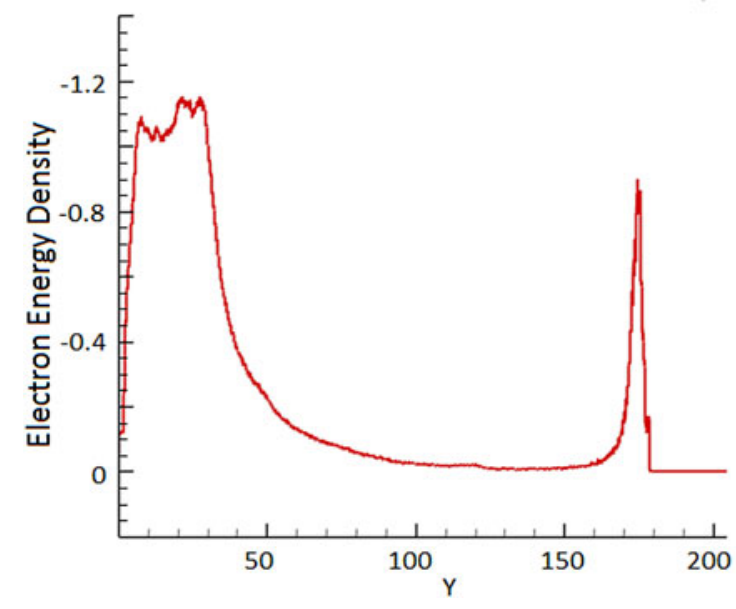

b)

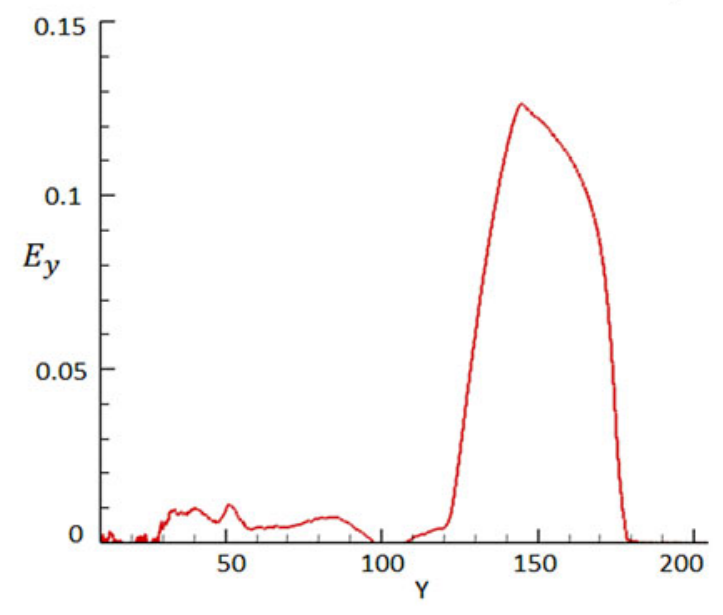

d)

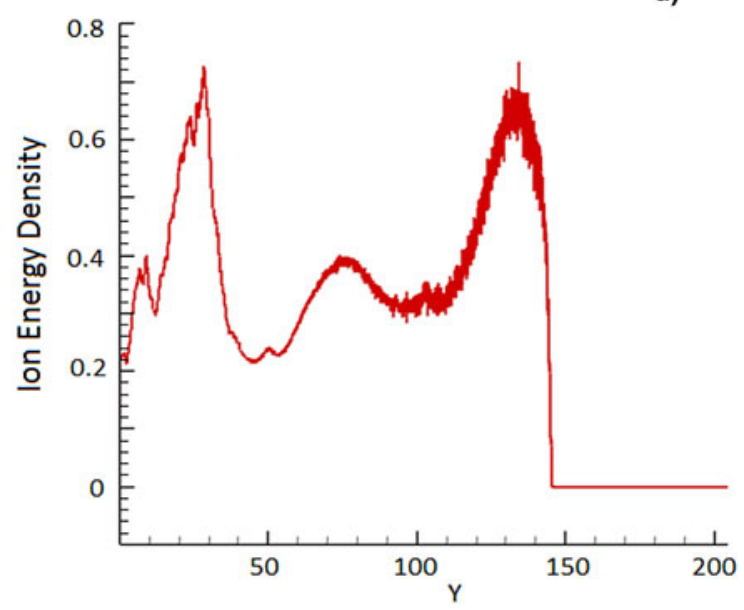

Fig. 7. The evolution of (a) magnetic vortex field $\left(\mathbf{B}_{z}\right)$ (b) longitudinal electric field $\left(\mathbf{E}_{\boldsymbol{y}}\right)$, (c) electron energy density, and (d) ion energy density; along the propagation direction ( $Y$-axis) followed by the laser field at $530 \mathrm{fsec}$ (just before where we get the maximum proton energy). The $Y$-axis are shown in units of laser wavelength. $\mathbf{B}_{z}$ and for $\mathbf{E}_{y}$ are expressed in the same units as in Figure 3 while the electron and ion energy densities are expressed in units of $n_{\mathrm{c}} m_{\mathrm{e}} c^{2}$.

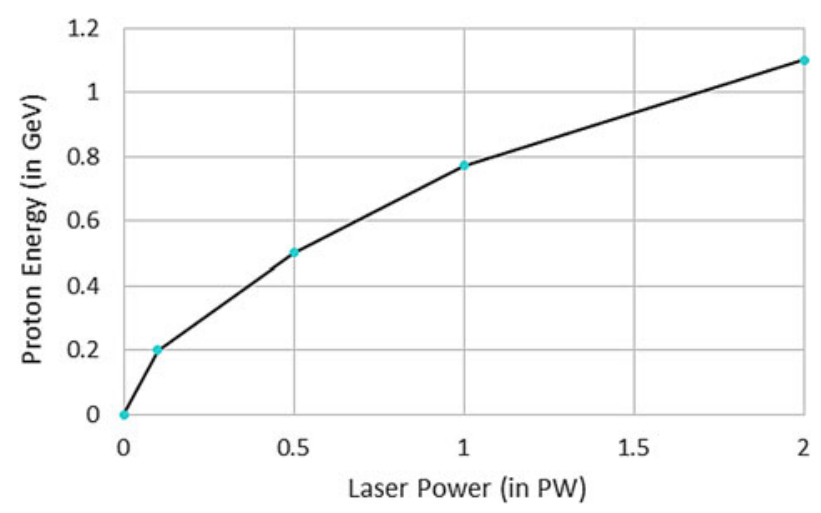

Fig. 8. The dependence of maximal proton energy on the laser power. The black line curve shows the maximum proton energy from simulation results, while the blue dot corresponds to the laser power where the simulation is performed.
$\mathrm{PW}$ range laser has repetition rate about $10 \mathrm{~Hz}$, it means that our laser will have at least ten times higher efficiency in the production of fast protons.

\section{CONCLUSION}

In conclusion, we demonstrated the acceleration of ions from near-critical density hydrogen plasma employing the ultrashort laser pulse by 3D PIC simulation. We obtained the maximum proton energy $\sim \mathrm{GeV}$ by employing the $2 \mathrm{PW} /$ 17 fs laser (http://www.eli-hu.hu/). The simulation results showed that an ion filament is formed on the axis of the plasma due to space charge attraction of high charge of the accelerated electron bunch. Significant part of the energetic ions forms a ring like spatial distribution. These protons are initially accelerated to sub $\mathrm{GeV}$ energy by a longitudinal 
electric field created by the time-varying magnetic field due to the return current of the lower energy electrons. We explored further that the MVA is effective for about $100 \mathrm{fs}$ (in case of our parameters) after the laser pulse exits from plasma-vacuum interface, further acceleration of protons occurred from the electrons co-moving with the laser pulse. The final proton energy was thus determined by MVA and the post acceleration. The magnetic field generated at the rear side of plasma-vacuum interface increases with the incident laser power for a given plasma density. We scaled the dependence of magnetic field on initial laser-plasma parameter. The analytical formulation shows the dependence of magnetic field on the laser power and the plasma density. 3D PIC simulation results show the ring shaped proton density distribution where the protons are accelerated along the laser axis with fairly low divergence accompanied by off-axis beam of ring-like shape. The target consideration of near critical gas plasma can be a better alternative to a thin solid foil because of its low cost, debris -free and can be operated at high repetition rate $(>10 \mathrm{~Hz})$ for efficient potential applications.

\section{ACKNOWLEDGEMENTS}

We performed 3D PIC simulation utilising the code PIConGPU [Bussmann et al. (2013)] in this research work. We acknowledge support of the Department of Information Services and Computing, Helmholtz-Zentrum Dresden-Rossendorf (HZDR), Germany; for providing access to the GPU Compute Cluster Hypnos. The authors also thank M. Bussmann, A. Huebl and the PIConGPU developer team for fruitful discussions regarding the simulation work; PIConGPU is developed and maintained by the Computational Radiation Physics Group at the Institute of Radiation Physics, HZDR.

\section{REFERENCES}

Amitani, H., Esirkepov, T., Bulanov, S., Nishihara, K., Kuznetsov, A. \& Kamenets, F. (2002). Accelerated dense ion filament formed by ultra-intense laser in plasma slab. AIP Conf. Proc. 611, 340-345.

Bake, M.A., Zhang, S., XIe, B.S., Hong, X.R. \& Wang, H.Y. (2012). Energy enhancement of proton acceleration in combinational radiation pressure and bubble by optimizing plasma density. Phys. Plasmas 19, 083103-083109.

BARAFFe, I. (2005). Structure and evolution of giant planets. Space Sci. Rev. 116, 67-76.

Borghesi, M., Mackinnon, A.J., Campbell, D.H., Hicks, D.G., Kar, S., Patel, P.K., Price, D., Romagnani, L., Schiavi, A. \& Willi, O. (2004). Multi-MeV proton source investigations in ultraintense laser-foil interactions. Phys. Rev. Lett. 92, 055003055006.

Borghesi, M., Sarri, G., Cecchetti, C.A., Kourakis, I., Hoarty, D., Stevenson, R.M., James, S., Brown, C.D., Hobbs, P., Lockyear, J., Morton, J., Willi, O., Jung, R. \& Dieckmann, M. (2010). Progress in proton radiography for diagnosis of ICF-relevant plasmas. Laser Part. Beams 28, 277-284.

Bulanov, S.V. \& Khoroshкov, V.S. (2002). Feasibility of using laser ion accelerators in proton therapy. Plasma Phys. Rep. 28, 453-456.
Bulanov, S.S., Bychenkov, V.Y., Chvykov, V., Kalinchenko, G., Litzenberg, D.W., Matsuoka, T., Thomas, A.G.R., Willingale, L., Yanovsky, V., Krushelnick, K. \& MaksimchuK, A. (2010a). Generation of $\mathrm{GeV}$ protons from $1 \mathrm{PW}$ laser interaction with near critical density targets. Phys. Plasmas 17, 043105-043112.

Bulanov, S.S., Esarey, E., Schroeder, C.B., Leemans, W.P., Bulanov, S.V., Margarone, D., Korn, G. \& Haberer, T. (2015). Helium-3 and helium-4 acceleration by high power laser pulses for hadron therapy. Phys. Rev. ST Accel. Beams 18, 061302-061307.

Bulanov, S.S., Litzenberg, D.W., Pirozhkov, A.S., Thomas, A.G.R., Willingale, L., Krushelnick, K. \& MaksimchuK, A. (2010b). Laser acceleration of protons from near critical density targets for application to radiation therapy, http://arxiv.org/ abs / 1007.3971

Bulanov, S.V., Dylov, D.V., EsiRkepov, T.Zh., Kamenets, F.F. \& Sokolov, D.V. (2005). Ion acceleration in a dipole vortex in a laser plasma corona. Plasma Phys. Rep. 31, 369-381.

Bussmann, M., Burau, H., Cowan, T.E., Debus, A., Huebl, A., Juckeland, G., Kluge, T., Nagel, W.E., Pausch, R., Schmitt, F., Schramm, U., Schuchart, J. \& Widera, R. (2013). Radiative Signatures of the Relativistic Kelvin-Helmholtz Instability, Proc. of the Int. Conf. on High Performance Comp., Networking, Storage and Anal. 5:1-5:12.

Burau, H. (2010). PIConGPU: A fully relativistic particle-in-cell code for a GPU cluster. IEEE Trans. Plasma Sci. 38, 2831-2839.

Cowan, T.E., Fuchs, J., Ruhl, H., Kemp, A., Audebert, P., Roth, M., Stephens, R., Barton, I., Blazevic, A., Brambrink, E., Cobble, J., Fernández, J., Gauthier, J.C., Geissel, M., Hegelich, M., KaAe, J., Karsch, S., Le Sage, G.P., Letzring, S., Manclossi, M., Meyroneinc, S., Newkirk, A., Pépin, H. \& Renard- LeGallouDEC, N. (2004). Ultralow emittance, multi-MeV proton beams from a laser virtual-cathode plasma accelerator. Phys. Rev. Lett. 92, 204801-204804.

Daido, H., Nishiuchi, M. \& Pirozhrov, A.S. (2012). Review of laser-driven ion sources and their applications. Rep. Prog. Phys. 75, 056401-056471.

Dyer, G.M., Bernstein, A.C., Cho, B.I., Osterholz, J., Grigsby, W., Dalton, A., Shepherd, R., Ping, Y., Chen, H., Widmann, K. \& Ditmire, T. (2008). Equation-of-state measurement of dense plasmas heated with fast protons. Phys. Rev. Lett. 101, 15002-15005.

ELI-ALPS: http://www.eli-alps.hu/

Esirkepov, T., Borghesi, M., Bulanov, S.V., Mourou, G. \& TaJima, T. (2004). Highly efficient relativistic-ion generation in the laserpiston regime. Phys. Rev. Lett. 92, 175003-175006.

Fiuza, F., Stockem, A., Boella, E., Fonseca, R.A., Silva, L.O., Haberberger, D., Tochitsky, S., Mori, W.B. \& Joshi, C. (2013). Ion acceleration from laser-driven electrostatic shocks. Phys. Plasmas 20, 056304-056311.

Fritzler, S., Malka, V., Grillon, G., Rousseau, J.P., and Burgy, F., Lefebrre, E., D’Humieres, E., McKenna, P. \& Ledingham, K.W.D. (2003). Proton beams generated with high-intensity lasers: Applications to medical isotope production. Appl. Phys. Lett. 83, 3039-3041.

Fuchs, J., Antici, P., D’Humières, E., Lefebvre, E., Borghesi, M., Brambrink, E., Cecchetti, C.A., Kaluza, M., Malka, V., Manclossi, M., Meyroneinc, S., Mora, P., Schreiber, J., Toncian, T., Pépin, H. \& Audebert, P. (2006). Laser-driven 
proton scaling laws and new paths towards energy increase. Nature Phys. 2, 48-54.

Fukuda, Y., Faenov, A.Ya., TAmpo, M., PikuZ, T.A., NaKamura, T., Kando, M., Hayashi, Y., Yogo, A., SaKaki, H., Kameshima, T., Pirozhrov, A.S., Ogura, K., Mori, M., Esirkepov, T.Z., Koga, J., Boldarev, A.S., Gasilov, V.A., Magunov, A.I., Yamauchi, T., Kodama, R., Bolton, P.R., Kato, Y., Tajima, T., Daido, H. \& Bulanov, S.V. (2009). Energy increase in multi-MeV ion acceleration in the interaction of a short pulse laser with a clustergas target. Phys. Rev. Lett. 103, 165002-165005.

Gaillard, S.A., Kluge, T., Flippo, K.A., Bussmann, M., Gall, B., Lockard, T., Geissel, M., Offermann, D.T., Schollmeier, M., Sentoku, Y. \& Cowan, T.E. (2011). Increased laser-accelerated proton energies via direct laser-light-pressure acceleration of electrons in microcone targets. Phys. Plasmas 18, 056710-056720.

GaO, L., WAng, H., Lin, C., Zou, Y. \& YAN, X. (2012). Efficient proton beam generation from a foam-carbon foil target using an intense circularly polarized laser. Phys. Plasmas 19, 083107-083110.

Gauthier, M., Levy, A., D'Humieres, E., Glesser, M., Albertazzi, B., Beaucourt, C., Brelli, J., Chen, S.N., Dervieux, V., Feugeas, J.L., Nicolai, P., Tikhonchuk, V., Pepin, H., Antici, P. \& Fuchs, J. (2014). Investigation of longitudinal proton acceleration in exploded targets irradiated by intense short-pulse laser. Phys. Plasmas 21, 013102-013112.

Gu, Y.J., Zhu, Z., Li, X.F., Yu, Q., Huang, S., Zhang, F., Kong, Q. \& Kawata, S. (2014). Stable long range proton acceleration driven by intense laser pulse with underdense plasmas. Phys. Plasmas 21, 063104-063109.

Haberberger, D., Tochitsky, S., Fiuza, F., Gong, C., Fonseca, R.A., Silva, L.O., Mori, W.B. \& Joshi, C. (2012). Collisionless shocks in laser-produced plasma generate monoenergetic highenergy proton beams. Nat. Phys. 8, 95-99.

Hatchett, S.P., Brown, C.G., Cowan, T.E., Henry, E.A., Johnson, J.S., Key, M.H., Koch, J.A., Langdon, A.B., Lasinski, B.F., LeE, R.W., Mackinnon, A.J., Pennington, D.M., Perry, M.D., Phillips, T.W., Roth, M., Sangster, T.C., Singh, M.C., Snavely, R.A., Stoyer, M.A., Wilks, S.C. \& Yasuike, K. (2000). Electron, photon, and ion beams from the relativistic interaction of Petawatt laser pulses with solid targets. Phys. Plasmas 7, 2076-2082.

Hegelich, B.M., Albright, B.J., Cobble, J., Flippo, K., Letzring, S., Paffett, M., Ruhl, H., Schreiber, J., Schulze, R.K. \& FERNÁNDEZ, J.C. (2006). Laser acceleration of quasimonoenergetic MeV ion beams. Nature 439, 441-444.

Henig, A., Kiefer, D., Markey, K., Gautier, D.C., Flippo, K.A., Letzring, S., Johnson, R.P., Shimada, T., Yin, L., Albright, B.J., Bowers, K.J., Fernández, J.C., Rykovanov, S.G., Wu, H.C., Zepf, M., Jung, D., Liechtenstein, V.Kh., Schreiber, J., Habs, D. \& Hegelich, B.M. (2009). Enhanced laser-driven ion acceleration in the relativistic transparency regime. Phys. Rev. Lett. 103, 045002-045005.

Hoffmann, D.H.H., Blazevic, A., Ni, P., Rosmej, O., Roth, M., Tahir, N.A., Tauschwitz, A., Udrea, S., Varentsov, D., WeYricj, K., and Maron, Y. (2005). Present and future perspectives for high energy density physics with intense heavy ion and laser beams. Laser Part. Beams 23, 47-53.

Jung, D., Albright, B.J., Yin, L., Gautier, D.C., Dromey, B., Shah, R., Palaniyappan, S., Letzring, S., Wu, H.-C., Shimada, T., Johnson, R.P., Habs, D., Roth, M., Fernandez, J.C. \& Heglich,
M. (2015). Scaling of ion energies in the relativistic-induced transparency regime. Laser Part. Beams 33, 695-703.

Jung, D., Yin, L., Albright, B.J., Gautier, D.C., Letzring, S., Dromey, B., Yeung, M., Hörlein, R., Shah, R. \& Palaniyappan, S. (2013). Efficient carbon ion beam generation from laser-driven volume acceleration. New J. Phys. 15, 023007023016 .

KaWata, S., Nagashima, T., Takano, M., IzUmiyama, T., Kamiyama, D., Barada, D., Kong, Q., Gu, Y.J., Wang, P.X., MA, Y.Y., Wang, W.M., Zhang, W., XIE, J., Zhang, H. \& DaI, D. (2014). Controllability of intense-laser ion acceleration. High Power Laser Sc. Eng. 2, 1-12.

Key, M.H., Akli, K., Beg, F., Chen, M.H., Chung, H.K., Freeman, R.R., Foord, M.E., Green, J.S., Gu, P., Gregori, G., Habara, H., Hatchett, S.P., Hey, D., Hill, J.M., King, J.A., Kodama, R., Koch, J.A., Lancaster, K., Lasinski, B.F., Langdon, B., MacKinnon, A.J., Murphy, C.D., Norreys, P.A., Patel, N., Patel, P., Pasley, J., Snavely, R.A., Stephens, R.B., Stoeckl, C., Tabak, M., Theobald, W., Tanaka, K., Town, R., Wilks, S.C., YabuUchi, Y.B. \& Zhang, B. (2006a). Study of electron and proton isochoric heating for fast ignition. J. Phys. IV France 133, 371-378.

Key, M.H., Freeman, R.R., Hatchett, S.P., MacKinnon, A.J., Patel, P.K., Snavely, R.A. \& Stephens, R.B. (2006b). Proton fast ignition. Fusion Sci. Technol. 49, 440-452.

KüHnel, M., Fernández, J.M., Tejeda, G., Kalinin, A., Montero, S. \& GRISENTI, R.E. (2011). Time-resolved study of crystallization in deeply cooled liquid parahydrogen. Phys. Rev. Lett. 106, 245301-245304.

Lemos, N., Martins, J.L., Dias, J.M., Marsh, K.A., PaK, A. \& Joshi, C. (2012). Forward directed ion acceleration in a LWFA with ionization-induced injection. J. Plasma Phys. 78, 327-331.

LoefFler, J.S. \& Durante, M. (2013). Charged particle therapy optimization, challenges and future directions. Nat. Rev. Clin. Oncol. 10, 411-424.

Macchi, A., Cattani, F., Liseykina, T.V. \& Cornolti, F. (2005). Laser acceleration of ion bunches at the front surface of overdense plasmas. Phys. Rev. Lett. 94, 165003-165006.

Mackinnon, A.J., Patel, P.K., Borghesi, M., Clarke, R.C., Freeman, R.R., Habara, H., Hatchett, S.P., Hey, D., Hicks, D.G., Kar, S., Key, M.H., King, J.A., Lancaster, K., Neely, D., Nikkro, A., Norreys, P.A., Notley, M.M., Phillips, T.W., Romagnani, L., Snavely, R.A., Stephens, R.B. \& Town, R.P.J. (2006). Proton radiography of a laser-driven implosion. Phys. Rev. Lett. 97, 045001-045004.

Matsukado, K., Esirkepov, T., Kinoshita, K., Daido, H., Utsumi, T., Li, Z., Fukumi, A., Hayashi, Y., Orimo, S., Nishiuchi, M., Bulanov, S.V., Tajima, T., Noda, A., Iwashita, Y., Shirai, T., Takeuchi, T., Nakamura, S., Yamazaki, A., Ikegami, M., Mihara, T., Morita, A., UesaKa, M., YoshiI, K., Watanabe, T., Hosokai, T., Zhidkov, A., Ogata, A., Wada, Y. \& Kubota, T. (2003). Energetic protons from a few-micron metallic foil evaporated by an intense laser pulse. Phys. Rev. Lett. 91, 215001-215004.

Muramatsu, M. \& Kitagawa, A. (2012). A review of ion sources for medical accelerators. Rev. Sci. Instrum. 83, 02B909-02B915.

Nakamura, T., Bulanov, S.V., Esirkepov, T.Zh. \& Kando, M. (2010). High-energy ions from near-critical density plasmas via magnetic vortex acceleration. Phys. Rev. Lett. 105, 135002-135005. 
Nickles, P.V., Ter-Avetisyan, S., Schnurer, M., Sokollik, T., Sandner, W., Schreiber, J., Hilscher, D., Jahnke, U., Andreev, A. \& TikhonchuK, V. (2007). Review of ultrafast ion acceleration experiments in laser plasma at Max Born Institute. Laser Part. Beams 25, 347-363.

Nycander, J. \& Isichenko, M.B. (1990). Motion of dipole vortices in a weakly inhomogeneous medium and related convective transport. Phys. Fluids B2, 2042-2047.

Palmer, C.A.J., Dover, N.P., Pogorelsky, I., Babzien, M., Dudnikova, G.I., Ispiriyan, M., Polyanskiy, M.N., Schreiber, J., Shrolnikov, P., Yakimenko, V. \& Najmudin, Z. (2011). Monoenergetic proton beams accelerated by a radiation pressure driven shock. Phys. Rev. Lett. 106, 014801-014804.

Robinson, A.P.L., Gibbon, P., ZepF, M., Kar, S., Evans, R.G. \& BelleI, C. (2009). Relativistically correct hole-boring and ion acceleration by circularly polarized laser pulses. Plasma Phys. Contr. Fus. 51, 024004-024017.

Robson, L., Simpson, P.T., Clarke, R.J., Ledingham, K.W.D., Lindau, F., Lundh, O., McCanny, T., Mora, P., Neely, D., Wahlström, C.G., Zepf, M. \& McKenna, P. (2007). Scaling of proton acceleration driven by petawatt-laser-plasma interactions. Nature Phys. 3, 58-62.

Roth, M., Cowan, T.E., Key, M.H., Hatchett, S.P., Brown, C., Fountain, W., Johnson, J., Pennington, D.M., Snavely, R.A., Wilks, S.C., Yasuike, K., Ruhl, H., Pegoraro, F., Bulanov, S.V., Campbell, E.M., Perry, M.D. \& Powell, H. (2001). Fast ignition by intense laser-accelerated proton beams. Phys. Rev. Lett. 86, 436-439.

Roth, M., Jung, D., Falk, K., Guler, N., Deppert, O., Devlin, M., Favalli, A., Fernandez, J., Gautier, D., Geissel, M., Haight, R., Hamilton, C.E., Hegelich, B.M., Johnson, R.P., Merrill, F., Schaumann, G., Schoenberg, K., Schollmeier, M., Shimada, T., Taddeucci, T., Tybo, J.L., Wagner, F., Wender, S.A.,
Wilde, C.H. \& Wurden, G.A. (2013). Bright laser-driven neutron source based on the relativistic transparency of solids. Phys. Rev. Lett. 110, 044802-044806.

Silva, L.O., Marti, M., Davies, J.R., Fonseca, R.A., Ren, C., Tsung, F.S. \& Mori, W.B. (2004). Proton shock acceleration in laser-plasma interactions. Phys. Rev. Lett. 9, 015002-015005.

Snavely, R.A., Key, M.H., Hatchett, S.P., Cowan, T.E., Roth, M., Phillips, T.W., Stoyer, M.A., Henry, E.A., SAngster, T.C., Singh, M.S., Wilks, S.C., MacKinnon, A., Offenberger, A., Pennington, D.M., Yasuike, K., Langdon, A.B., Lasinski, B.F., Johnson, J., Perry, M.D. \& Campbell, E.M. (2000). Intense high-energy proton beams from petawatt-laser irradiation of solids. Phys. Rev. Lett. 85, 2945-2948.

Wilks, S.C., Kruer, W.L., TABAK, M. \& LANGdon, A.B. (1992). Absorption of ultra-intense laser pulses. Phys. Rev. Lett. 69, 1383-1386.

Wilks, S.C., Langdon, A.B., Cowan, T.E., Roth, M., Singh, M., Hatchett, S., Key, M.H., Pennington, D., MacKinnon, A. \& SNAVElY, R.A. (2001). Energetic proton generation in ultraintense laser-solid interactions. Phys. Plasmas 8, 542-549.

YaO, W., Li, B., CaO, L., Zheng, F., Huang, T., XiaO, H. \& Skoric, M.M. (2014). Generation of monoenergetic proton beams by a combined scheme with an overdense hydrocarbon target and an underdense plasma gas irradiated by ultra-intense laser pulse. Laser Part. Beams 32, 583-589.

Yin, L., Albright, B.J., Bowers, K.J., Jung, D., FernándeZ, J.C. \& Hegelich, B.M. (2011). Three-dimensional dynamics of breakout afterburner ion acceleration using high-contrast shortpulse laser and nanoscale targets. Phys. Rev. Lett. 107, 045003- 045006.

Yin, L., Albright, B.J., Hegelich, B.M. \& Fernández, J.C. (2006), $\mathrm{GeV}$ laser ion acceleration from ultrathin targets: The laser break-out afterburner. Laser Part. Beams 24, 291-298. 